\title{
Particle Ratios, Equilibration, and the QCD Phase Boundary
}

\author{
P. Braun-Munzinger \\ Gesellschaft für Schwerionenforschung, D-64291 Darmstadt, Germany
}

\section{J. Stachel}

Physikalisches Institut der Universität Heidelberg, D 69120 Heidelberg, Germany

\begin{abstract}
.
We discuss the status of thermal model descriptions of particle ratios in central nucleus-nucleus collisions at ultra-relativistic energy. An alternative to the "Cleymans-Redlich" interpretation of the freeze-out trajectory is given in terms of the total baryon density. Emphasis is placed on the relation between the chemical equilibration parameters and the QCD phase boundary. Furthermore, we trace the essential difference between thermal model analyses of data from collisions between elementary particles and from heavy ion collisions as due to a transition from local strangeness conservation to percolation of strangeness over large volumes, as occurs naturally in a deconfined medium. We also discuss predictions of the thermal model for composite particle production.
\end{abstract}

The thermal model has been used very successfully to reproduce and (for RHIC data even) predict particle ratios measured in central collisions of heavy nuclei at ultrarelativistic energy [1, 2, 3, 4, 5, 6. In particular, the predictions for the RHIC data are very close to the experimental observations: for a temperature of $\mathrm{T}=174 \mathrm{MeV}$ and a baryon chemical potential of $\mu_{b}=46 \mathrm{MeV}$ all available data measured near central rapidity are reproduced with a $\chi^{2}$ of 5.7 for 7 effective degrees of freedom [6]. We note that these calculations are performed under the condition of full strangeness equilibration and global strangeness conservation, i.e. the flow of strangeness into and out of the central rapidity slice is assumed to cancel. For an update on the inclusion of the most recent data see [7], where it is demonstrated that even the $\phi / \mathrm{h}^{-}$ratio is well described with the same set of parameters.

These analyses yield a parameter pair $\left(\mathrm{T}, \mu_{b}\right)$ for each energy where data have been obtained. The current state of affairs is described in Fig. 11. In this plot of temperature vs chemical potential, the location of the phase boundary between hadronic matter and the quark-gluon plasma is indicated by the dotted and dashed lines, taken from the latest calculations within the framework of lattice QCD [8] and from a bag model equation of state [9]. The data points in this figure represent chemical freeze-out points determined by a thermal analysis [1, 2, 3, 6, 10 of hadron multiplicities measured in central $\mathrm{Pb}+\mathrm{Pb}$ or $\mathrm{Au}+\mathrm{Au}$ collisions at various beam energies at the SIS, AGS, SPS and RHIC accelerator facilities. The line connecting the data points demonstrates, in the framework of the hadron resonance gas model used in [3], that chemical freeze-out appears to take place at constant total baryon 


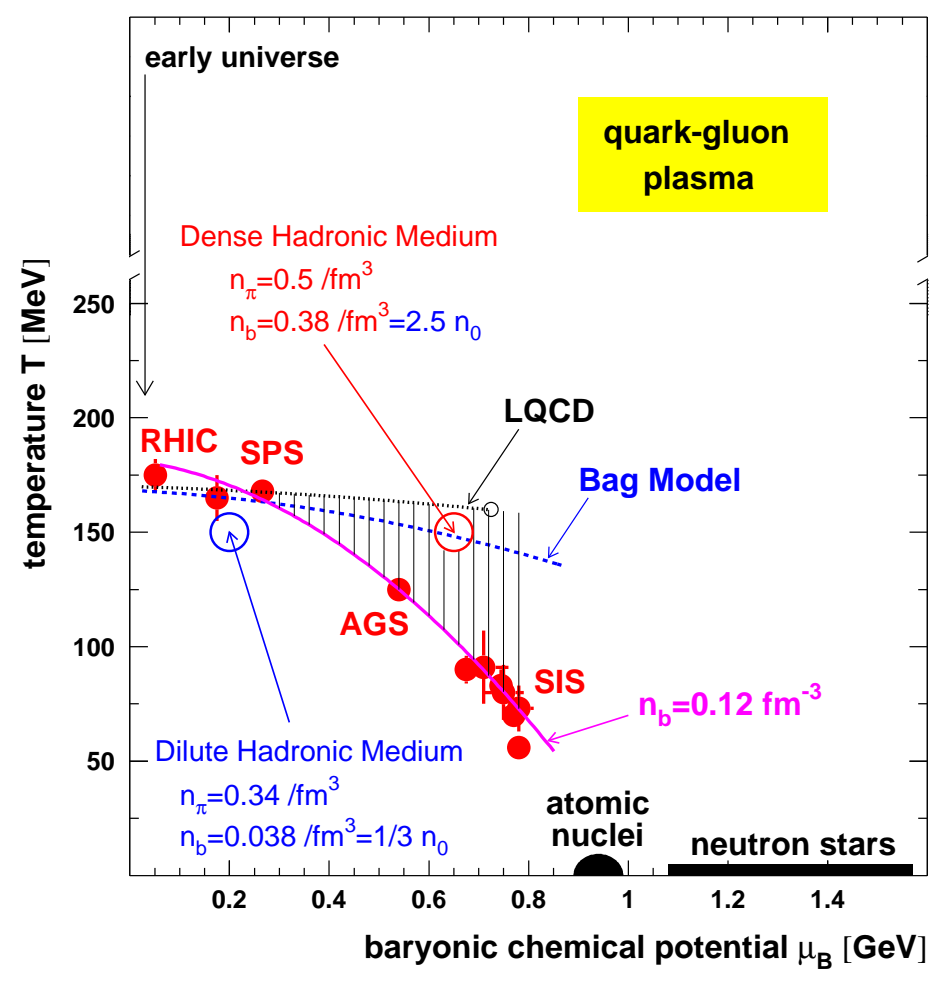

Figure 1. The QCD Phase Diagram in the context of the current knowledge about relativistic nuclear collisions. For details see text.

plus anti-baryon density $\mathrm{n}_{b}=0.12 / \mathrm{fm}^{3}$. The so determined freeze-out line crosses the calculated phase boundary roughly at full SPS energy.

We note that this interpretation of the freeze-out trajectory differs significantly from that of [5], where chemical freeze-out is assumed to take place at constant total energy per particle $\langle E\rangle /\langle N\rangle \approx 1 \mathrm{GeV}$. To us the constant total baryon density scenario is more appealing as it implies a physical picture: chemical freeze-out takes place at a critical baryon density through baryon-baryon and baryon mesoninteractions. The absolute value of the critical baryon density $\mathrm{n}_{b}=0.12 / \mathrm{fm}^{3}$ depends of course on the fact that, in [3], a repulsive hadron-hadron interaction is implemented via an excluded volume.

The fact that the chemical freeze-out points closely approach the calculated QCD phase boundary lends strong support to the interpretation 9 , 11, 12, 13, 14] that the matter produced in nuclear collisions at SPS and RHIC energies was first thermalized in the deconfined quark-gluon plasma phase and subsequently expanded through the phase boundary into a thermal gas of (weakly interacting) hadrons.

Furthermore, as is illustrated in Fig. 1, the hadronic matter underneath the phase boundary for values of the baryon chemical potential $\mu_{b}<300 \mathrm{MeV}$, roughly corresponding to full SPS energy and higher, is always dilute: the total baryon density 
never exceeds that of normal nuclear matter. We, consequently, do not expect a strong influence on the chemical freeze-out scenario due to changed hadron masses in the medium, if SPS and RHIC energies are considered. Furthermore, even if one includes very strong medium effects such as in the chiral model of [15], the chemical freeze-out still occurs close to the phase boundary. In the analysis of [15] the phase boundary is at $\mathrm{T}_{c}=154 \mathrm{MeV}$, while the chiral model analysis of the data yields $\mathrm{T}=144 \mathrm{MeV}$.

It is only at lower beam energies, between 5 and $50 \mathrm{GeV} /$ nucleon, that one can identify between chemical freeze-out and the phase boundary a phase of dense hadronic matter, shown by the hatched area in Fig. 11. It is near these beam energies, i.e. close to $30 \mathrm{GeV} /$ nucleon, where also the relative strangeness content of the hadronic medium is maximum 16.

This interpretation calls into question the widely accepted belief that the enhanced yield of low mass dileptons observed at full SPS energy by the CERES collaboration [17, 18] is mainly due to interactions of the $\rho$-meson with baryons [19].

At $40 \mathrm{GeV} /$ nucleon beam energy, analysis 20] of the (still preliminary) hadron yields from the NA45, NA49, and NA57 collaborations leads to freeze-out parameters of $\mathrm{T} \approx 140 \mathrm{MeV}$ and $\mu_{b} \approx 400 \mathrm{MeV}$. This $\mu_{b}$ value is very close to the value of about $420 \mathrm{MeV}$ obtained from the phenomenological parameterization provided by equation 1 in [16] at $40 \mathrm{GeV} /$ nucleon. At this energy, the CERES collaboration has recently provided first, preliminary results on low-mass di-electrons [21, 22]. Again, an enhancement relative to the yield expected from standard hadronic sources is observed. In fact, the observed enhancement seems to increase from $2.7 \pm 0.65$ at 160 $\mathrm{GeV} /$ nucleon to $4.5 \pm 1.2$ at $40 \mathrm{GeV} /$ nucleon. Whether this is due to the increase, at 40 compared to $160 \mathrm{GeV} /$ nucleon, of the baryon density between chemical freeze-out and the phase boundary is an interesting speculation. However, if Fig. 1 represents reality, this increase is only slight, and the real effect should develop at around 20 $\mathrm{GeV} /$ nucleon. Whether the few days of running currently planned at the CERN SPS in 2002 at 30 and $20 \mathrm{GeV} /$ nucleon will lead to further insights into this intriguing question is unclear at present.

We note that the planned new GSI accelerator facility [23] is tailored to explore in detail the interesting region between 5 and $30 \mathrm{GeV} /$ nucleon, where a dense hadronic medium is expected between the QCD phase boundary and chemical freeze-out.

The above discussed connection between the QCD phase boundary and the observed chemical freeze-out points is sometimes called into question 24, 25] since hadron production in $\mathrm{e}^{+} \mathrm{e}^{-}$and pp or $\mathrm{p} \overline{\mathrm{p}}$ can also be described in thermal model 26] yielding a (apparently universal) temperature $\mathrm{T}_{e} \approx 170 \mathrm{MeV}$. While the fact that $\mathrm{T}_{e}$ is close to $\mathrm{T}$ values determined for heavy ion collisions at top SPS and RHIC energies (corresponding to relatively small values of $\mu_{b}$ ) might indeed reflect the fundamental hadronization scale of QCD, we note that there is an essential difference between thermal descriptions of central heavy ion collisions and elementary particle reactions. As discussed, particle densities and their ratios can be, for heavy ion collisions at full AGS energy and higher, well described in the grand-canonical ensemble using the particle density $\mathrm{n}_{\mathrm{gc}}$. In contrast, for a description of particle production in elementary particle collisions, local quantum number conservation needs to be taken into account explicitly, leading, for the production of particles with strangeness $n$ to a canonical suppression factor

$$
F_{c}=\frac{I_{n}\left(x_{1}\right)}{I_{0}\left(x_{1}\right)},
$$


with which the grand-canonical densities have to be multiplied to obtain the results in the canonical ensemble. Here, $I_{n}$ denotes a modified Bessel function of degree $n$ and the parameter $x_{1}=2 \mathrm{Vn}_{\mathrm{gc}}$ depends explicitly on the volume $\mathrm{V}$ over which quantum number conservation is enforced [27]. For $x_{1}>>1, \frac{I_{n}\left(x_{1}\right)}{I_{0}\left(x_{1}\right)} \rightarrow 1$, the volume drops out and the grand-canonical limit applicable to ultra-relativistic nuclear collisions is recovered. For $x_{1}<<1, \frac{I_{n}\left(x_{1}\right)}{I_{0}\left(x_{1}\right)} \rightarrow \frac{\left(1 / 2 \cdot x_{1}\right)^{n}}{n !}$. This case, appropriate for the analysis of $\mathrm{e}^{+} \mathrm{e}^{-}$and pp or $\mathrm{p} \overline{\mathrm{p}}$ collisions, implies that densities of particles with strangeness $n$ contain a quantum number dependent volume $\mathrm{V}$. Hence, the $\mathrm{K} / \pi$ ratios are proportional to $\mathrm{V}$, the $\Omega / \pi$ ratios proportional to $\mathrm{V}^{3}$. For the description of elementary particle collisions $\mathrm{V}=\mathrm{V}_{0} \approx 7 \mathrm{fm}^{3}[27$ is required, i.e. strangeness is conserved within approximately the volume of a nucleon. Moving to an analysis of heavy ion collisions one finds [16, 27] that $\mathrm{V}=\mathrm{V}_{0} \cdot \mathrm{N}_{\text {part }} / 2$ where $\mathrm{N}_{\text {part }}$ is the number of participants. Already in central Au-Au collisions at SIS energies this "correlation volume" V exceeds $1000 \mathrm{fm}^{3}$. The required volume increases further as the beam energy is increased, until the grand-canonical limit is reached at full SPS energy.

This implies that, in central nucleus-nucleus collisions at ultra-relativistic energies, strangeness percolates freely over volumes of thousands of $\mathrm{fm}^{3}$ ! At top SPS and RHIC energies it is natural to conclude that the percolation has its origin in the quark-gluon phase, lending further strong support to the interpretation above that the "coincidence" between experimentally determined chemical freeze-out points and the calculated phase boundary implies that a deconfined phase was produced in such collisions. An interesting question is what leads to the percolation at SIS energies, where the freeze-out points are far away from where we think the phase boundary is. We speculate that, in this case, the percolation takes place in the high density hadronic phase indicated by the hatched area in Fig. 1 .

An often overlooked aspect of the thermal model is the possibility to compute also the yields of composite particles. For example, the $d / p$ and $\bar{d} / \bar{p}$ ratios measured at SPS and AGS energies are well reproduced with the same parameters which are used to describe baryon and meson ratios [1, 2, 3]. Furthermore, the AGS E864 collaboration has recently published [28] yields for composite particles (light nuclei) produced in central $\mathrm{Au}-\mathrm{Au}$ collisions at AGS energy near mid-rapidity. In this investigation, an exponential decrease of composite particle yield with mass is observed, implying a penalty factor of about 48 for each additional nucleon. Extrapolation of the data to large transverse momentum values reduces this penalty factor to about 26 , principally because of transverse flow. In the thermal model, this penalty factor can be easily derived. In the relevant Boltzmann approximation, we obtain

$$
R_{p} \approx \exp \frac{m \pm \mu_{b}}{T},
$$

where $\mathrm{m}$ is the nucleon mass and the negative sign applies for matter, the positive for anti-matter. Small corrections due to the spin degeneracy and the $\mathrm{A}^{3 / 2}$ term in front of the exponential in the Boltzmann formula for particle density are neglected. Using the freeze-out parameters $\mathrm{T}=125 \mathrm{MeV}$ and $\mu_{b}=540 \mathrm{MeV}$ appropriate for AGS energy [1. 3] we calculate $\mathrm{R}_{p} \approx 23$, in close agreement with the data for the production of light nuclei. We also note that the anti-matter yields measured by the E864 collaboration [29] yield penalty factors of about $2 \cdot 10^{5}$, again close to the predicted value of $1.3 \cdot 10^{5}$.

This rather quantitative agreement between measured relative yields for composite particles and thermal model predictions provides some confidence in the 
Particle Ratios, Equilibration, and the QCD Phase Boundary

Table 1. Produced number of nonstrange and strange clusters and of strange quark matter per central $\mathrm{Au}+\mathrm{Au}$ collisions at AGS energy, calculated in a thermal model for two different temperatures, baryon chemical potential $\mu_{b}=0.54 \mathrm{GeV}$ and strangeness chemical potential $\mu_{s}$ such that overall strangeness is conserved. The coalescence model predictions are from Table 2 of [31.

\begin{tabular}{|c|c|c|c|}
\hline \multirow[b]{2}{*}{ Particles } & \multicolumn{2}{|c|}{ Thermal Model } & \multirow[b]{2}{*}{ Coalescence Model } \\
\hline & $T=.120 \mathrm{GeV}$ & $T=.140 \mathrm{GeV}$ & \\
\hline $\mathrm{d}$ & 15 & 19 & 11.7 \\
\hline $\mathrm{t}+{ }^{3} \mathrm{He}$ & 1.5 & 3.0 & 0.8 \\
\hline$\alpha$ & 0.02 & 0.067 & 0.018 \\
\hline$H_{0}$ & 0.09 & 0.15 & 0.07 \\
\hline $5 \mathrm{H}$ & $3.5 \cdot 10^{-5}$ & $2.3 \cdot 10^{-4}$ & $4 \cdot 10^{-4}$ \\
\hline $\begin{array}{l}{ }_{6}{ }_{\Delta \Lambda} \\
\Delta \mathrm{He}\end{array}$ & $7.2 \cdot 10^{-7}$ & $7.6 \cdot 10^{-6}$ & $1.6 \cdot 10^{-5}$ \\
\hline$\Xi^{0} \Lambda \Lambda \mathrm{He}$ & $4.0 \cdot 10^{-10}$ & $9.6 \cdot 10^{-9}$ & $4 \cdot 10^{-8}$ \\
\hline${ }_{10}^{10} \mathrm{St}^{-8}$ & $1.6 \cdot 10^{-14}$ & $7.3 \cdot 10^{-13}$ & \\
\hline${ }_{1}^{12} \mathrm{St}^{-9}$ & $1.6 \cdot 10^{-17}$ & $1.7 \cdot 10^{-15}$ & \\
\hline${ }_{1}^{1} \mathrm{St}^{-11}$ & $6.2 \cdot 10^{-21}$ & $1.4 \cdot 10^{-18}$ & \\
\hline${ }_{0}^{1} 6 \mathrm{St}^{-13}$ & $2.4 \cdot 10^{-24}$ & $1.2 \cdot 10^{-21}$ & \\
\hline $20 \mathrm{St}^{-16}$ & $9.6 \cdot 10^{-31}$ & $2.3 \cdot 10^{-27}$ & \\
\hline
\end{tabular}

predictions for yields of exotic objects produced in central nuclear collisions. We briefly comment here on the results of a 1995 analysis [30].

In this investigation, the production probabilities for exotic strange objects and, in particular, for strangelets were computed in the thermal model. The results are reproduced in Table 1 for temperatures relevant for beam energies between 10 and $40 \mathrm{GeV} /$ nucleon. We first note that predictions of the thermal model and, where available, the coalescence model of 31 agree (maybe surprisingly) well. Secondly, inspection of Table 1 also shows that, in future high statistics experiments which will be possible at the planned new GSI facility [23], multi-strange objects such as ${ }_{=}^{7} \Lambda \Lambda$ He should be experimentally accessible with a planned sensitivity of about $10^{-13}$ per central collision in a years running, should they exist and be produced with thermal yields. Investigation of yields of even the lightest conceivable strangelets will be difficult, though.

In summary, we have discussed the surprising success of the thermal model to reproduce particle yields in central nucleus-nucleus collisions at ultra-relativistic energies. These investigations yield chemical freeze-out parameters which approach the phase boundary for energies at and above full SPS energy. The implications of this observation are, as discussed above, that the matter produced in nuclear collisions at SPS and RHIC energies was most likely first thermalized in a deconfined quarkgluon plasma phase and subsequently expanded through the phase boundary into a gas of hadrons. In a detailed discussion of various areas of the phase diagram we have identified a zone of dense hadronic matter between the expected phase boundary and chemical freeze-out which can be studied experimentally with beams in the energy region $10<\mathrm{E}_{l a b} / \mathrm{A}<40 \mathrm{GeV}$. Furthermore, we have traced the essential difference between thermal model analyses of data from collisions between elementary particles and from heavy ion collisions as due to a transition from local strangeness conservation to percolation of strangeness over large volumes, as occurs naturally in a deconfined medium. Finally, a discussion of composite particle production has emphasized the 
success of the thermal model also in this area and indicated possibilities to study experimentally also rather exotic and interesting multi-strange hadrons.

\section{References}

[1] Braun-Munzinger P, Stachel J, Wessels J P and Xu N 1995 Phys. Lett. B 34443

[2] Braun-Munzinger P, Stachel J, Wessels J P and Xu N 1996 Phys. Lett. B 3651

[3] Braun-Munzinger P, Heppe I and Stachel J 1999 Phys. Lett. B 46515

[4] Becattini F, Gazdzicki M and Sollfrank J 1998 Eur. J. Phys. C 5143

[5] Cleymans J and Redlich K 1999 Phys. Rev. C 60054908

[6] Braun-Munzinger P. Magestro D, Redlich K and Stachel J 2001 Phys. Lett. B 51841 (Preprint hep-ph/0105229)

[7] Magestro D 2001 these proceedings (Preprint hep-ph/0112178)

[8] Fodor Z and Katz S D 2001 Preprint hep-lat/0106002

[9] Braun-Munzinger P and Stachel J 1996 Nucl. Phys. A 606320

Note that, in Fig. 11, the dashed line is calculated with a rescaled bag constant such that $\mathrm{T}_{c}$ $=167 \mathrm{MeV}$ for $\mu_{b}=0$.

[10] Braun-Munzinger P and Stachel J 1998 Nucl. Phys. A 638 3c

[11] Stock R 1999 Phys. Lett. B 456277

[12] Heinz U 1999 Nucl. Phys. A 661 140c

[13] Stachel J 1999 Proc. INPC, Paris, August 1998 Nucl. Phys. A 654 119c

[14] Braun-Munzinger P 2000 Proc. PANIC, Uppsala, June 1999 Nucl. Phys. A 663-664 183

[15] Zschiesche D et al 2001 Nucl. Phys. A $68134 \mathrm{c}$

[16] Braun-Munzinger P, Cleymans J, Oeschler H and Redlich K 2001 Nucl. Phys. A in print (Preprint hep-ph/0106066)

[17] Agakichiev G et al CERES collaboration 1995 Phys. Rev. Lett. 751272

[18] Agakichiev G et al CERES collaboration 1998 Phys. Lett. B 422405

[19] Rapp R and Wambach J 2000 Adv. Nucl. Phys. 251 and references therein

[20] Schmitz W 2001 Dissertation, University of Heidelberg

[21] Damjanovic S et al CERES collaboration 2001 Proc. Int. Europhysics Conf., Budapest, Oct. 2001

(Preprint nucl-ex/0111009)

[22] Filimonov Ket al. Proc. IN PC 2001 Conference, Berkeley, August 2001 (Preprint nucl-ex/0109017)

[23] An International Accelerator facility for Beams of Ions and Antiprotons, Conceptual Design Report, GSI, Nov. 2001.

[24] Specht H J 2001 Proc. QM2001 conference, Stony Brook, Jan. 2001 Nucl. Phys. A in print (Preprint nucl-ex/0111011)

[25] Nagle J L 2001 Proc. International Nuclear Physics Conference (INPC 2001), Berkeley, California. July 2001 (Preprint nucl-ex/0109016)

[26] Becattini F and Heinz U 1997 Z. Phys. C 76269 (Preprint hep-ph/9702274)

See also: Becattini F Preprint hep-ph/9701275

[27] Redlich K 2001 Preprint hep-ph/0111383

Redlich K and Tounsi A Preprint hep-ph/0111261

[28] Armstrong T A et al E864 collaboration 2000 Phys. Rev. C 61064908

[29] Armstrong T A et al E864 collaboration 2000 Phys. Rev. Lett. 852685

[30] Braun-Munzinger P and Stachel J 1995 J. Phys. G: Nucl. Part. Phys. 21 L17

[31] Baltz A J et al 1994 Phys. Lett. B 3257

Note that eq. (5) of this manuscript contains an error which is corrected in our Table 1 